\title{
Analytical solutions to rocket performance with an arbitrary thrust programme having constant thrust inclination with respect to the horizon in vacuum
}

\author{
S N MAITRA \\ Department of Mathematics, National Defence Academy, PO NDA, \\ Khadakwasla, Pune 411 023, India \\ MS received 16 July 1986; revised 9 March 1988
}

\begin{abstract}
The present investigations pertain to rocket performance due to manoevering in a vacuum with any arbitrary thrust inclined at a constant angle with respect to the horizon. Considering the variable thrust either as a function of time, or mass ratio, it is established that dynamical equations projected on the horizontal and vertical directions can be solved analytically; closed-form solutions to the overall range, endurance and final or impact velocity arising out of both burning and coasting phases can be achieved depending on the nature of the thrust function. However, in the first section the thrust inclination is made equal to $90^{\circ}$, in so much so that the path of the rocket becomes vertical evoking the determination, for this case, of its peak velocity and altitude. Finally, this analysis leads us to conclude that with the same propellant mass ratio, initial mass, and thrust inclination to the horizon, a programme of any greater thrust is superior to that of any lower thrust:

Rocket performance with an arbitrary thrust programme of greater thrust function evolves greater peak altitude for vertical path, and greater overall range, albeit shorter burnout range, for the curvilinear path.
\end{abstract}

Keywords. Rocket performance; arbitrary thrust programme; constant thrust inclination; propellant mass; burnout conditions.

\section{Introduction}

Miele (1962, pp. 335-355) analysed the motion of a rocket flown in vacuum, with constant thrust inclination with respect to the horizon, for three particular thrust programmes: (i) constant thrust, (ii) constant acceleration, and (iii) zero thrust. The present analyses have been carried out in the context of an "unbounded thrust" which either increases or decreases monotonically and lasts till the exhaustion of the entire propellant mass. The author's previous analysis (Maitra 1980) is related to the variable thrust programming given by $\tau=\tau_{i} \mu^{n}$ where the instantaneous thrust-toweight ratio is proportional to the $n$th power of the mass ratio, $n$ being a constant.

A list of symbols is given at the end of the paper. 
The earlier analyses carried out by Miele (1962, pp. 335-355) and the present author (Maitra 1980) differ from the present one by introducing in the latter the thrust as any arbitrary function of time or mass ratio, which depends upon the law of variation of thrust assigned as a particular thrust programme to a space mission. Let the thrust be considered as a function of mass ratio so that

or

$$
T=m_{i} g f(\mu) \text {, }
$$

$$
f(\mu)=T / m_{i} g, \text { or } f(\mu)=\tau \mu,
$$

and initially when

$$
\mu=\mu_{i}=1, f(1)=\tau_{i} .
$$

In a solid propellant rocket, it is possible, with suitable grain designs, to obtain a wide variety of pre-programmed thrust laws along the burning time. Variable thrust can also be attained in liquid-propellant rockets. Choosing the thrust as an arbitrary function of the mass ratio in this paper, first the peak altitude and the time to ascend it in the case of a vertically ascending rocket, and second, the range, endurance and final velocity in the case of a rocket flown with constant thrust inclination with respect to the horizon in a vacuum have been determined analytically. The equations of motion of a rocket in a vertical plane as given by Miele (1962, pp. 335-355) are

$$
\begin{aligned}
& \dot{X}-V_{x}=0, \\
& \dot{h}-V_{h}=0, \\
& T \cos \delta-m \dot{V}_{x}=0, \\
& T \sin \delta-m\left(g+\dot{V}_{h}\right)=0, \\
& \dot{m}+\beta=0, \\
& T=\beta V_{E} .
\end{aligned}
$$

\section{Vertically ascending path}

By introducing the non-dimensional parameters

$$
\theta=t g / V_{E}, h^{\prime}=h g / V_{E}^{2}, u=V / V_{E}, \mu=m / m_{i}, \tau=\beta V_{E} / m g,
$$

in the thrust programme represented by (1), imposing $\delta=90^{\circ}$ in (2) and selecting $\mu$ as the new independent variable, the equations of motion of the vertically ascending rocket in vacuum are transformed into

$$
\begin{aligned}
& \left(\mathrm{d} h^{\prime} / \mathrm{d} \mu\right)+[u / f(\mu)]=0, \\
& (\mathrm{~d} u / \mathrm{d} \mu)+(1 / \mu)-[1 / f(\mu)]=0, \\
& (\mathrm{~d} \theta / \mathrm{d} \mu)+[1 / f(\mu)]=0 .
\end{aligned}
$$

Assuming the initial conditions that

$$
\theta_{i}=u_{i}=h_{i}^{\prime}=0, \mu_{i}=1,
$$

(4) can be solved yielding the following burnout conditions

$$
\theta_{c}=-\int_{1}^{\mu_{c}}[\mathrm{~d} \mu / f(\mu)],
$$




$$
\begin{aligned}
& u_{c}=-\log \mu_{c}+\int_{1}^{\mu_{c}}[\mathrm{~d} \mu / f(\mu)], \\
& h_{c}^{\prime}=\int_{1}^{\mu_{c}}[\log \mu / f(\mu)] \mathrm{d} \mu-\int_{1}^{\mu_{c}}\left[\int_{1}^{\mu}\left(\mathrm{d} \mu^{\prime} / f\left(\mu^{\prime}\right)\right][\mathrm{d} \mu / f(\mu)] .\right.
\end{aligned}
$$

The peak altitude and time of its ascent at the end of the coasting phase, are obtained by employing (7) and (8) and then (6) and (7):

$$
\begin{aligned}
h_{f}^{\prime}= & h_{c}^{\prime}+\left(u_{c}^{2} / 2\right), \quad\left(\mu_{c}=\mu_{f}\right), \\
h_{f}^{\prime}= & \int_{1}^{\mu_{c}} \frac{\log \mu}{f(\mu)} \mathrm{d} \mu-\int_{1}^{\mu_{c}}\left[\int_{1}^{\mu} \frac{\mathrm{d} \mu^{\prime}}{f\left(\mu^{\prime}\right)}\right] \frac{\mathrm{d} \mu}{f(\mu)}+\frac{\log ^{2} \mu_{c}}{2}+ \\
& +\frac{1}{2}\left[\int_{1}^{\mu_{c}} \frac{\mathrm{d} \mu}{f(\mu)}\right]^{2}-\left(\log \mu_{c}\right) \int_{1}^{\mu_{c}} \frac{\mathrm{d} \mu}{f(\mu)} .
\end{aligned}
$$

Integrating by parts the first and second integrals of the right hand side;

$$
h_{f}^{\prime}=\log ^{2} \mu_{f}[(1 / 2)-(1 / K)] \text {, }
$$

where

$$
1 / K=\left(1 / \log ^{2} \mu_{f}\right) \int_{1}^{\mu_{c}}\left\{(1 / \mu) \int_{1}^{\mu}\left[\mathrm{d} \mu^{\prime} / f\left(\mu^{\prime}\right)\right]\right\} \mathrm{d} \mu,
$$

whereas the value of the same $K$ in the previous paper (Maitra 1980) is

$$
\left(-\tau_{i} n^{2} \log ^{2} \mu_{f}\right) /\left(1-\mu_{f}^{-n}-n \log \mu_{f}\right) \text {, for } \tau=\tau_{i} \mu^{n}
$$

which can also be obtained herein upon substitution in (10)

$$
f(\mu)=\tau \mu=\tau_{i} \mu^{n+1} .
$$

Given the thrust function $f(\mu)$, the peak altitude $h_{f}^{\prime}$ can be computed analytically. Second, the time of ascent is

$$
\theta_{f}=\theta_{c}+u_{c}=-\log \mu_{f} .
$$

Obviously, the results obtained so far in the present design will reduce to those associated with (i) constant thrust, (ii) constant acceleration programmes, and (iii) variable thrust programme, respectively, where (i) $\tau \mu=f(\mu)=\tau_{i}$, (ii) $f(\mu)=\mu \tau_{i}$ and (iii) $f(\mu)=\tau \mu=\tau_{i} \mu^{n+1}$, on account of (4).

\section{Range and endurance with constant thrust inclination}

Making use of the dimensionless variables

$$
X^{\prime}=X g / V_{E}^{2}, \quad \phi=V_{x} / V_{E}, \quad \psi=V_{h} / V_{E},
$$

together with some conditions of (3), initial conditions

$$
X^{\prime}=h^{\prime}=\phi_{i}=\psi_{i}=u_{i}=\theta_{i}=0, \quad \mu_{i}=1,
$$


and the thrust function prescribed by (1) and also by taking $\mu$ as the independent variable, equations (2) become

$$
\begin{aligned}
& \mathrm{d} X^{\prime} / \mathrm{d} \mu=-\phi / f(\mu), \\
& \mathrm{d} h^{\prime} / \mathrm{d} \mu=-\psi / f(\mu), \\
& \mathrm{d} \phi / \mathrm{d} \mu=-\cos \delta / \mu, \\
& \mathrm{d} \psi / \mathrm{d} \mu=[1 / f(\mu)]-(\sin \delta / \mu), \\
& \mathrm{d} \theta / \mathrm{d} \mu=-1 / f(\mu) .
\end{aligned}
$$

Applying the initial conditions, (14) can be solved to obtain the following burnout conditions:

$$
\begin{aligned}
& \theta_{c}=-E\left(\mu_{c}\right), \quad \phi_{c}=-\cos \delta \log \mu_{c}, \quad \psi_{c}=E\left(\mu_{c}\right)-\sin \delta \log \mu_{c}, \\
& X_{c}^{\prime}=\cos \delta F\left(\mu_{c}\right), \quad h_{c}^{\prime}=-S\left(\mu_{c}\right)+\sin \delta F\left(\mu_{c}\right),
\end{aligned}
$$

where

$$
\begin{aligned}
& E\left(\mu_{c}\right)=\int_{1}^{\mu_{c}}[\mathrm{~d} \mu / f(\mu)], \quad F\left(\mu_{c}\right)=\int_{1}^{\mu_{c}}[\log \mu / f(\mu)] \mathrm{d} \mu, \\
& S\left(\mu_{c}\right)=\int_{1}^{\mu_{c}}[E(\mu) \mathrm{d} \mu / f(\mu)], \quad E(\mu)=\int_{1}^{\mu}\left[\mathrm{d} \mu^{\prime} / f\left(\mu^{\prime}\right)\right] .
\end{aligned}
$$

After reaching the burnout conditions (15), the rocket is allowed to travel without burning of propellant, i.e., along a coasting arc leading to the final conditions:

$$
\begin{aligned}
& \mu_{c}=\mu_{f}, \\
& \phi_{c}=\phi_{f}, \\
& \psi_{f}+\theta_{f}=\psi_{c}+\theta_{c}, \\
& X_{f}^{\prime}-\phi_{f} \theta_{f}=X_{c}^{\prime}-\phi_{c} \theta_{c}, \\
& \psi_{f}^{2} / 2=h_{c}^{\prime}+\left(\psi_{c}^{2} / 2\right),
\end{aligned}
$$

and consequently the coasting range is given by

$$
X_{f}^{\prime}-X_{c}^{\prime}=\phi_{c}\left[\psi_{c}+\left(2 h_{c}^{\prime}+\psi_{c}^{2}\right)^{\frac{1}{2}}\right] \geqslant 0 \text {. }
$$

By virtue of (15) to (18), the overall range attained by the rocket ensures

$$
\begin{aligned}
X^{\prime}= & X_{c}+\phi_{c}\left[\psi_{c}+\left(2 h_{c}^{\prime}+\psi_{c}^{2}\right)^{\frac{1}{t}}\right] \\
= & F\left(\mu_{c}\right) \cos \delta-\log \mu_{f} \cos \delta\left\{E\left(\mu_{c}\right)-\sin \delta \log \mu_{f}+\right. \\
& \left.+\left[\left[E\left(\mu_{c}\right)-\sin \delta \log \mu_{f}\right]^{2}+2\left[F\left(\mu_{c}\right) \sin \delta-S\left(\mu_{c}\right)\right]\right]^{\frac{1}{2}}\right\} .
\end{aligned}
$$

Now an interesting relation between $S\left(\mu_{c}\right)$ and $E\left(\mu_{c}\right)$ is given below:

$$
S\left(\mu_{c}\right)=\int_{1}^{\mu_{c}}\left[\int_{1}^{\mu}\left[\mathrm{d} \mu^{\prime} / f\left(\mu^{\prime}\right)\right]\right][\mathrm{d} \mu / f(\mu)]=\int_{1}^{\mu_{c}}[E(\mu) / f(\mu)] \mathrm{d} \mu .
$$

Integrating by parts and using the fundamental theorem of integral calculus

$$
S\left(\mu_{c}\right)=\left[E(\mu) \int[\mathrm{d} \mu / f(\mu)]\right]_{1}^{\mu_{c}}-\int_{1}^{\mu_{c}}\left[\int[\mathrm{d} \mu / f(\mu)]\right][\mathrm{d} \mu / f(\mu)],
$$


$1 / f(\mu)$ is a continuous function so that $\mathrm{d}\{E(\mu)\} / \mathrm{d} \mu=1 / f(\mu)$. Owing to the last equation of set (16), $E(\mu)=\varepsilon(\mu)-\varepsilon(1)$ letting $\varepsilon(\mu)=\int \mathrm{d} \mu / f(\mu)$ so that $E\left(\mu_{c}\right)=\varepsilon\left(\mu_{c}\right)-\varepsilon(1)$ and $E(1)=0$ for $1>\mu \geqslant \mu_{c}$. In view of this,

$$
\begin{aligned}
S\left(\mu_{c}\right) & =E\left(\mu_{c}\right) \cdot \varepsilon\left(\mu_{c}\right)-\int_{1}^{\mu_{c}}\{E(\mu)+\varepsilon(1)\}[\mathrm{d} \mu / f(\mu)] \\
& =E\left(\mu_{c}\right) \cdot \varepsilon\left(\mu_{c}\right)-\int_{1}^{\mu_{c}}[E(\mu) / f(\mu)] \mathrm{d} \mu-\varepsilon(1) \int_{1}^{\mu_{c}}[\mathrm{~d} \mu / f(\mu)] \\
& =E\left(\mu_{c}\right)\left\{\varepsilon\left(\mu_{c}\right)-\varepsilon(1)\right\}-S\left(\mu_{c}\right) \\
& =E^{2}\left(\mu_{c}\right)-S\left(\mu_{c}\right) \\
\text { or, } 2 S\left(\mu_{c}\right) & =E^{2}\left(\mu_{c}\right) .
\end{aligned}
$$

By the use of (19), the foregoing expression for the overall range is rewritten as

Further,

$$
\begin{aligned}
X_{f}^{\prime}= & \cos \delta \log ^{2} \mu_{c}\left\{\sin \delta-\left[\left(\left(E / \mu_{c}\right) \log \mu_{c}-F\left(\mu_{c}\right)\right) / \log ^{2} \mu_{c}\right]+\right. \\
& \left.+\left\{\sin \delta\left[\sin \delta-2\left[\left(E\left(\mu_{c}\right) \log \mu_{c}-F\left(\mu_{c}\right)\right) / \log ^{2} \mu_{c}\right]\right]\right\}^{\frac{1}{2}}\right\}
\end{aligned}
$$

$$
E\left(\mu_{c}\right) \log \mu_{c}-F\left(\mu_{c}\right)=\log \mu_{c} \int_{1}^{\mu_{c}}[\mathrm{~d} \mu / f(\mu)]-\int_{1}^{\mu_{c}}[\log \mu / f(\mu)] \mathrm{d} \mu .
$$

Integrating the second integral by parts and using the integrals used for the derivation of (19),

$$
\begin{aligned}
& E\left(\mu_{c}\right) \log \mu_{c}-F\left(\mu_{c}\right) \\
& =\log \mu_{c} E\left(\mu_{c}\right)-\log \mu_{c} \cdot \varepsilon\left(\mu_{c}\right)+\int_{1}^{\mu_{c}}[\varepsilon(\mu) / \mu] \mathrm{d} \mu \\
& =\log \mu_{c} \cdot \varepsilon(1)+\int_{1}^{\mu_{c}}[(E(\mu)+\varepsilon(1)) / \mu] \mathrm{d} \mu \\
& =\int_{1}^{\mu_{c}}[E(\mu) / \mu] \mathrm{d} \mu=\int_{1}^{\mu_{c}}\left\{\int_{1}^{\mu}\left[\mathrm{d} \mu^{\prime} / f\left(\mu^{\prime}\right)\right]\right\}(\mathrm{d} \mu / \mu) .
\end{aligned}
$$

Finally, the overall range comprising the burning and coasting phases, is formulated as

$$
\begin{aligned}
& X_{f}^{\prime}=\cos \delta \log ^{2} \mu_{f}\left\{\sin \delta-(1 / K)+[\sin \delta(\sin \delta-(2 / K))]^{\frac{1}{2}}\right\} \\
& \left(\mu_{c}=\mu_{f}\right)
\end{aligned}
$$

where

$$
1 / K=\int_{1}^{\mu_{c}}\left[\int_{1}^{\mu}\left(\mathrm{d} \mu^{\prime} / f\left(\mu^{\prime}\right)\right)\right](\mathrm{d} \mu / \mu) / \log ^{2} \mu_{f}=\int_{1}^{\mu_{c}}[(E(\mu) / \mu) \mathrm{d} \mu] / \log ^{2} \mu_{f}
$$


which is the same function of the propellant mass as encountered in the preceding section.

Again,

$$
\int_{1}^{\mu_{c}}[E(\mu) / \mu] \mathrm{d} \mu=-\int_{\mu_{c}}^{1}[E(\mu) / \mu] \mathrm{d} \mu=\int_{\mu_{c}}^{1}\left[\int_{\mu}^{1}\left(\mathrm{~d} \mu^{\prime} / f\left(\mu^{\prime}\right)\right)\right] \mathrm{d} \mu / \mu>0,
$$

for

$$
\mu_{c}<\mu<1 \text {, as } \int_{\mu}^{1}\left[\mathrm{~d} \mu^{\prime} / f\left(\mu^{\prime}\right)\right]>0 \text { for all } \mu<\mu^{\prime}<1,
$$

which is again due to

$$
1 / f\left(\mu^{\prime}\right)>0 \text { i.e., } f(\mu)>0 \text { for } \mu<1 \text {. }
$$

In consequence of (23) and (24), $1 / K$ is a positive quantity for all $\mu_{c}<1$ and tends to zero as $\mu_{c} \rightarrow 1$. Equation (22) reveals that the overall range $X_{f}^{\prime}$ increases as $K$ increases subject to the constraint

$$
1 / K \leqslant \sin \delta / 2
$$

whose physical significance has already been explained in the earlier paper (Maitra 1980).

In order to get at the idea of variation of $K$ with the change of thrust function, let $K_{1}$ and $K_{2}$ correspond, respectively, to the thrust functions $T_{1}=m_{i} g f_{1}(\mu)$ and $T_{2}=m_{i} g f_{2}(\mu)$ so that

$$
\begin{aligned}
1 / K_{1} & =\int_{1}^{\mu_{c}}\left[\int_{1}^{\mu}\left[\mathrm{d} \mu^{\prime} / f_{1}\left(\mu^{\prime}\right)\right]\right](\mathrm{d} \mu / \mu) / \log ^{2} \mu_{f}, \\
1 / K_{2} & =\int_{1}^{\mu_{c}}\left\{\left[\int_{1}^{\mu}\left[\mathrm{d} \mu^{\prime} / f_{2}\left(\mu^{\prime}\right)\right]\right](\mathrm{d} \mu / \mu)\right\} / \log ^{2} \mu_{f},
\end{aligned}
$$

which from the inequality theory of integral calculus implies that if

$$
f_{1}(\mu)>f_{2}(\mu) \text {, then } K_{1}>K_{2} \text { for } \mu_{c} \leqslant \mu \leqslant 1 \text {. }
$$

For the same initial thrust and weight or equivalently for the same initial thrust-toweight ratio, $f_{1}(1)=f_{2}(1)$.

Hence, if $T_{1}(\mu)>T_{2}(\mu)$ for $\mu_{c} \leqslant \mu \leqslant 1$, then the overall range associated with the former thrust programme, because of the fact that $K_{1}>K_{2}$ and (22), becomes greater than that associated with the latter, for the same propellant mass ratio, initial mass and constant thrust inclination to the horizon. In other words, a greater thrust programme as defined above leads to greater overall range with the same three conditions. As a consequence of $(22)$ to (26), the necessary and sufficient conditions for a thrust programme $T_{1}(\mu)$ to yield the overall range greater than or equal to that by another thrust programme $T_{2}(\mu)$ is $1 / K_{1} \leqslant 1 / K_{2}$, with the given conditions mentioned already. In view of (9) a similar observation can be made as regards the peak altitude achieved by a rocket flown vertically. 
In consideration of some equations of the sets (17) and (18), the time of flight, i.e., endurance of the rocket can be evaluated as

$$
\theta_{f}=\theta_{c}+\left[\left(X_{f}^{\prime}-X_{c}^{\prime}\right) / \phi_{c}\right]=-\log \mu_{f}\left\{\sin \delta+\left[\sin \delta(\sin \delta-(2 / K)]^{1 / 2}\right\}\right.
$$

Equations (22) and (27) confirm that range and endurance increase with the increase of propellant mass and thrust inclination to the horizon, respectively. As in the previous analysis, with given propellant mass ratio and initial mass there exists in this analysis a thrust inclination $\delta$ which maximizes the overall range so that

$$
\left[X_{f}^{\prime}\right]_{\max }=\cos \delta \log ^{2} \mu_{f} / 2 \sin ^{3} \delta
$$

where

$$
K=2 \sin ^{3} \delta /\left(2 \sin ^{2} \delta-1\right) .
$$

The optimum thrust inclination that can be determined by solving (23) and (29) graphically or otherwise, is substituted in (28) to find the maximum range with given thrust law $f(\mu)$. Because of (27) and (29), the time taken to acquire this maximum range or in other words the endurance for the maximum range turns out to be

$$
\left[\theta_{f}\right]_{\text {max range }}=-\log \mu_{f} / \sin \delta, \quad\left(\mu_{f}<1\right) .
$$

However, combining (28) and (30), the maximum range can be expressed in terms of the endurance and propellant mass ratio as

$$
X_{f}^{\prime}=\left[-\theta_{f}^{2}\left(\theta_{f}^{2}-\log ^{2} \mu_{f}\right)^{1 / 2}\right] / 2 \log \mu_{f}, \quad\left(\mu_{f}<1\right) .
$$

\section{Discussion and conclusion}

That the present paper is regarded as a generalisation of the author's earlier work (Maitra 1980) can be verified by means of (1) and (23). For the variable thrust programme $\tau=\tau_{i} \mu^{n}(n \neq-1)$ in consequence of $(1), f(\mu)=\tau_{i} \mu^{n+1}$ is to be put in (23) so as to get $K=\left(-\tau_{i} n^{2} \log ^{2} \mu_{f}\right) /\left[\left(1-\mu_{f}^{-n}\right)+\log \mu_{f}^{-n}\right]$ which is the same as obtained in the earlier analysis (Maitra 1980). The values of $K$ obtained in the same process for constant thrust and constant acceleration programmes in fact agree with those in Miele's analysis. Take an example of another variable thrust programme

$$
T=T_{i} e^{\alpha(1-\mu)},(\alpha \neq 0),
$$

or equivalently, $f(\mu)=\tau \mu=\tau_{i} e^{\alpha(1-\mu)}$; in this case (23) shows

$$
K=\left(-\alpha e^{\alpha} \tau_{i} \log ^{2} \mu_{f}\right) /\left[Y(\alpha)+e^{\alpha} \log \mu_{f}-Y\left(\alpha \mu_{f}\right)\right],
$$

where

$$
Y(\alpha)=\int_{-\infty}^{\alpha}\left(e^{x} \mathrm{~d} x\right) / x
$$

is called the Euler's or exponential integral and can be expanded in the following series: 


$$
\begin{aligned}
& Y(\alpha)=\gamma_{E}+\log \alpha+\sum_{n=1}^{n} \alpha^{n} / n ! n . \\
& \left(\gamma_{E}=\text { Euler's constant }\right)
\end{aligned}
$$

It can be noticed that this thrust programme becomes a constant thrust programme if the characteristic parameter $\alpha$ tends to zero and as such

$$
\begin{aligned}
& \operatorname{Lim}_{\alpha \rightarrow 0} K=\operatorname{Lim}_{\alpha \rightarrow 0}\left\{\left(-\alpha e^{\alpha} \tau_{i} \log ^{2} \mu_{f}\right) /\left[Y(\alpha)+e^{\alpha} \log \mu_{f}-Y\left(\alpha \mu_{f}\right)\right]\right\}(0 / 0), \text { by (35), } \\
& \operatorname{Lim}_{\alpha \rightarrow 0} K=\left(-\tau_{i} \log ^{2} \mu_{f}\right) /\left[\left(1-\mu_{f}\right)+\log \mu_{f}\right],
\end{aligned}
$$

which is the value of $K$ as obtained for the constant thrust programme in the analysis (Miele 1962, pp. 335-355; Maitra 1980).

For realizing the prescribed thrust programme $T=T(\mu)$ in practice, the time history of the thrust function is required; so by means of the last equation of set (14), or the first of (15), it can be made a function of time. For instance, in the analysis (Maitra 1980) for the thrust programme $\tau=\tau_{i} \mu^{n}(n \neq 0, n=-1)$ where the time and mass ratio $\mu$ are related by $\theta=-\left(1 / n \tau_{i}\right)\left(1-\mu^{-n}\right)$, the thrust expressed as a function of time $\theta$ is

$$
T=m_{i} g \tau_{i}\left(n \tau_{i} \theta+1\right)^{-[1+(1 / n)]} .
$$

Obviously in the case of constant thrust $n=-1$, so that

$$
T=m_{i} g \tau_{i},
$$

and in the case of constant acceleration (Miele 1962, pp. 335-355), the thrust becomes

$$
T=m_{i} g \tau_{i} e^{-\tau_{i} \theta}=T_{i} e^{-\tau_{i} \theta},
$$

or otherwise from the preceding result taking $n \rightarrow 0$,

$$
T=m_{i} g \tau_{i} \lim _{n \rightarrow 0}\left[\left(n \tau_{i} \theta+1\right) /\left(n \tau_{i} \theta+1\right)^{1 / n}\right] .
$$

By the use of the formula $\operatorname{Lim}_{x \rightarrow 0}(1+x a)^{\frac{1}{x}}=e^{a}$,

$$
T=m_{i} g \tau_{i} e^{-\tau_{i} \theta}=\tau_{i} e^{-\tau_{i} \theta} .
$$

If the thrust is prescribed as a function of time, i.e., $T=T(\theta)$, in this situation also the complete solutions can be worked out to the rocket motion under the present quest, taking time as the independent variable. Considering the thrust law $f(\theta)=T(\theta) / m_{i} g$, and the time $\theta$ as the independent variable, (2) together with the dimensionless parameters (12) and some of (3) give rise to

$$
\begin{aligned}
& \mathrm{d} X^{\prime} / \mathrm{d} \theta=\phi, \quad \mathrm{d} h^{\prime} / \mathrm{d} \theta=\psi, \\
& \mathrm{d} \phi / \mathrm{d} \theta=[\cos \delta f(\theta)] / \mu, \quad \mathrm{d} \psi / \mathrm{d} \theta=-1+(\sin \delta / \mu) f(\theta), \quad \mathrm{d} \theta / \mathrm{d} \mu=-1 / f(\theta),
\end{aligned}
$$

which, when integrated with the help of the initial conditions (13), yield the following 
burnout conditions:

$$
\begin{aligned}
\mu_{c}= & 1-\int_{0}^{\theta_{c}} f(\theta) \mathrm{d} \theta, \quad \phi_{c}=-\cos \delta \log \left\{1-\int_{0}^{\theta_{c}} f(\theta) \mathrm{d} \theta\right\}, \\
\psi_{c}= & -\theta_{c}-\sin \delta \log \left\{1-\int_{0}^{\theta_{c}} f(\theta) \mathrm{d} \theta\right\} \\
h_{\mathrm{c}}^{\prime}= & \frac{-\theta_{c}^{2}}{2}-\sin \delta\left\{\theta_{c} \log \left[1-\int_{0}^{\theta_{c}} f(\theta) \mathrm{d} \theta\right]+\right. \\
& \left.+\int_{0}^{\theta_{c}}\left[\theta f(\theta) \mathrm{d} \theta /\left(1-\int_{0}^{\theta} f\left(\theta^{\prime}\right) \mathrm{d} \theta^{\prime}\right)\right]\right\},
\end{aligned}
$$

(Integrating by parts)

$$
X_{c}^{\prime}=-\cos \delta\left\{\theta_{c} \log \left[1-\int_{0}^{\theta_{c}} f(\theta) \mathrm{d} \theta\right]+\int_{0}^{\theta_{c}}\left[\theta f(\theta) \mathrm{d} \theta /\left(1-\int_{0}^{\theta} f\left(\theta^{\prime}\right) \mathrm{d} \theta^{\prime}\right)\right]\right\},
$$

where $0<\theta^{\prime}<\theta<\theta_{c}$.

The results (37) can be combined with the final conditions (17) so as to derive an expression for the overall range in terms of the burning time $\theta_{c}$ :

$$
X_{f}^{\prime}=\cos \delta \log ^{2}\left\{1-\int_{0}^{\theta_{c}} f(\theta) \mathrm{d} \theta\right\}\left\{\sin \delta-\frac{1}{K}+[\sin \delta(\sin \delta-2 / K)]^{\frac{1}{2}}\right\}
$$

where

$$
1 / K=\left\{\int_{0}^{\theta_{c}}\left[\theta f(\theta) \mathrm{d} \theta /\left(1-\int_{0}^{\theta} f\left(\theta^{\prime}\right) \mathrm{d} \theta^{\prime}\right)\right]\right\} /\left[\log ^{2}\left(1-\int_{0}^{\theta_{c}} f(\theta) \mathrm{d} \theta\right)\right] .
$$

Equations (38) and (39) can also be deduced upon substitution of the first condition of (37) and that too without subscript $c$, into (22) and (23). by

From the second and third equations of the set (15) the burnout velocity is given

$$
\begin{aligned}
u_{c} & =\left(\phi_{c}^{2}+\psi_{c}^{2}\right)^{1 / 2} \\
& =\left\{E^{2}\left(\mu_{c}\right)+\log ^{2} \mu_{c}-2 \sin \delta \int_{1}^{\mu_{k}}[\mathrm{~d} \mu / f(\mu)] \log \mu_{c}\right\}^{\frac{1}{2}},
\end{aligned}
$$

which, with given propellant mass ratio $\mu_{c}$, increases with the decrease of thrust inclination $\delta$ where $0<\delta<\pi / 2$. The impact or final velocity that is obtained at the end of the coasting flight is determined by using the second and third equations of the set (15), the second and fifth of (17), and then the fifth of (15) as

$$
u_{f}=-\{1-(2 / K) \sin \delta\}^{1 / 2} \log \mu_{f}, \quad\left(\mu_{c}=\mu_{f}\right)
$$


which also increases with the decrease of thrust inclination, because of (23) and (24).

Although the thrust inclination to the horizon is held constant in the present operation, the flight-path angle does not remain constant. The second and third equations of set (15) give the path inclination at burnout position so that

$$
\tan \gamma_{c}=\psi_{c} / \phi_{c}=\left[\sin \delta \log \mu_{c}-E\left(\mu_{c}\right)\right] /\left(\cos \delta \log \mu_{c}\right) .
$$

The instantaneous path inclination is obtained by deleting subscript $c$ from (42). Equation (42) indicates that for given propellant mass ratio the flight path angle increases as the thrust inclination increases.

For $0<\psi<\pi / 2$, by considering (42)

$$
\tan \delta / \sec \delta>E\left(\mu_{c}\right) / \log \mu_{c}>0,
$$

implying that

$$
\mathrm{d}(\tan \psi) / \mathrm{d} \delta=\sec ^{2} \delta-\left[E\left(\mu_{c}\right) / \log \mu_{c}\right] \sec \delta \tan \delta>\sec ^{2} \delta-\tan ^{2} \delta=1 .
$$

By the use of the second and fifth equations of set (17) and the fifth of (16) along with (23), the final path inclination $\gamma_{f}$ is given by

$$
\tan ^{2} \gamma_{f}=\psi_{f}^{2} / \phi_{f}^{2}=\tan ^{2} \delta-(2 / K) \tan \delta \sec \delta>0,
$$

which ensures that with $0<\delta<\pi / 2$,

$$
\mathrm{d}\left(\tan ^{2} \gamma_{f}\right) / \mathrm{d} \delta>2 \tan \delta \sec ^{2} \delta-(\tan \delta / \sec \delta)\left(\sec ^{3} \delta+\tan ^{2} \delta \sec \delta\right)=\tan \delta>0 .
$$

Therefore, the greater the thrust inclination, the greater is the final path inclination or angle of impact. What is more important from the attitude control point of view is the inclination of the thrust vector with respect to the tangent to the flight path, which is in general aligned with the axis of the rocket in motion. This angle of inclination, say $v$, is called thrust angle of attack that can be disposed, as a consequence of (42), in the form

$$
\begin{aligned}
\tan v & =\tan (\gamma-\delta) \\
& =-E(\mu) /[(\log \mu) \sec \delta-E(\mu) \tan \delta] .
\end{aligned}
$$

The thrust angle of attack can also be made an explicit function of time to foresee the thrust direction in this design of flight:

$$
\tan v=\theta /\left\{\log \left[E^{-1}(-\theta)\right] \sec \delta+\theta \tan \delta\right\}
$$

where $\mu$ is evaluated as a function of time $\theta$ from the first equation of set (15) such that $\mu=E^{-1}(-\theta)$. Obviously the thrust angle of attack at the burnout point is acquired from (43), or (43a) wherein subscript $c$ is to be attached to ' $\gamma$ ' and ' $\theta$ '. In other words, if the thrust be assigned as a function of time, then in view of the second and third equations of the set (37) the thrust angle of attack is spelled out to be 


$$
\tan \gamma=\theta /\left\{\sec \delta \log \left[1-\int_{0}^{\theta} f\left(\theta^{\prime}\right) \mathrm{d} \theta^{\prime}\right]+\theta \tan \delta\right\} .
$$

In order to execute appropriate attitude control for such a type of thrust programme, the thrust inclination with respect to the tangent to the flight path (i.e to the direction of flight) is made known a priori by means of (43a) or (43b) which gives the time history of variation of the thrust direction with respect to the vehicle axis - when the latter is collinear with the tangent to the flight path, till the burning phase is over.

The effects of greater thrust programme on the burnout parameters given by (15) can be studied as follows. Because of the first equations of the sets (15) and (16), burning time is less for the greater thrust programme. For given thrust inclination and propellant mass ratio, the horizontal component of the velocity remains unchanged, whatever may be the thrust law, because of the second equation of the set (15).

The fourth equation of (14) is set forth owing to (1) as

$$
\begin{aligned}
\mathrm{d} \psi / \mathrm{d} T & =\mathrm{d} \psi /\left\{m_{i} g \mathrm{~d}[f(\mu)]\right\}=\left(1 / m_{i} g\right)[(1 / f(\mu)-(\sin \delta / \mu)]\{\mathrm{d} \mu / \mathrm{d}[f(\mu)]\} \\
& =\text { positive or negative, depending on } \mu, f(\mu) \text { and } \delta
\end{aligned}
$$

Moreover, $\mathrm{d} f(\mu) / \mathrm{d} \mu$ is positive or negative according to whether the thrust is monotonic decreasing (regressive) or increasing (progressive). Otherwise, if the thrust programme is given by

$$
f(\mu)=\mu / \sin \delta,
$$

then $\psi=\psi_{i}=0$; in that case because of no vertical component of velocity, the vehicle executes a horizontal path till the end of propellant burning. While studying the performance of this typical thrust programme leading to horizontal motion during the burning period, because of (44a) the burnout conditions (15) reduce to the following form;

$$
\begin{aligned}
& \theta_{c}=-\sin \delta \log \mu_{c}, \quad \phi_{c}=-\cos \delta \log \mu_{c} \\
& h_{c}^{\prime}=h_{i}^{\prime}, \psi_{c}=0 \\
& X_{c}^{\prime}=\int_{1}^{\mu_{c}} \cos \delta(\log \mu / \mu) \sin \delta \mathrm{d} \mu=(\cos \delta \sin \delta / 2) \log ^{2} \mu_{c}
\end{aligned}
$$

Here the initial conditions have to be changed slightly in comparison to the previous ones by assuming the launching altitude $h_{i}$. The combination of (45) with (18) leads to the overall range

$$
\begin{aligned}
& X_{f}^{\prime}=(\cos \delta \sin \delta / 2) \log ^{2} \mu_{f}-\cos \delta \log \mu_{c} \cdot\left(2 h_{i}\right)^{1 / 2}, \\
& \mathrm{~d} X_{f}^{\prime} / \mathrm{d} \delta=0 \text { gives } \sin \delta=\left[-\lambda+\left(\lambda^{2}+2\right)^{1 / 2}\right] / 2,(0<\delta<\pi / 2), \\
& \text { where } \quad 0<\lambda=-\left(2 h_{i}\right)^{1 / 2} / \log \mu_{f}=\cos 2 \delta / 2 \sin \delta ;\left(\mu_{c}=\mu_{f}\right) \\
& \text { Also, } \mathrm{d}^{2} X_{f}^{\prime} / \mathrm{d} \delta^{2}=-\sin 2 \delta \log ^{2} \mu_{f}+\left(2 h_{i}^{\prime}\right)^{1 / 2} \cos \delta \log \mu_{f}
\end{aligned}
$$

By the use of the preceding relationship; $(0<\delta<\pi / 2)$

$$
\left.\left(\mathrm{d}^{2} x_{f}^{\prime} / \mathrm{d} \delta^{2}\right)_{\delta=\hat{\delta}}=-\log ^{2} \mu_{f}(\cos \hat{\delta} / 2 \sin \hat{\delta})\left(1+2 \sin ^{2} \hat{\delta}\right)\right]<0 \text {, by }(47 \mathrm{a})
$$


Again for $0<\sin \delta<1$, (47) gives $0<\lambda$. Hence, with given launching altitude and propellant mass ratio subject to the restriction $(47 a)$, there exists a thrust inclination $\delta$, because of (47) and (48), maximizing the overall range. However, the maximum range can be expressed in the following form as a result of the second part (47a) of (47) used in (46):

$$
\left[X_{f}^{\prime}\right]_{\max }=\left(\cos ^{3} \delta / 2 \sin \delta\right) \log ^{2} \mu_{f}
$$

Now (44) reveals that if $f(\mu)>\mu / \sin \delta$ and at the same time the thrust monotonically increases, resulting in a curvilinear trajectory convex upwards, then for the same thrust inclination and instantaneous propellant mass ratio $\mu$ the horizontal component of the velocity increases with the intake of higher thrust programme. For more clarity let subscripts 1 and 2 correspond to the burnout parameters evolved by the thrust programmes $T_{1}(\mu)$ and $T_{2}(\mu)$ respectively so that in consideration of (1), the third of (15), and the first of (16), one gets

$$
\psi_{c_{1}}=\int_{1}^{\mu_{c}}\left[\mathrm{~d} \mu / f_{1}(\mu)\right]-\sin \delta \log \mu_{c} \text { and } \psi_{c_{2}}=\int_{1}^{\mu_{c}}\left[\mathrm{~d} \mu / f_{2}(\mu)\right]-\sin \delta \log \mu_{c},
$$

which, by the theory of integral calculus, implies that if $f_{1}(\mu)>f_{2}(\mu)$, then $\psi_{c_{1}}>\psi_{c_{2}}$ and also in general $\psi_{1}>\psi_{2}$ for $\mu_{c} \leqslant \mu<1$ and $0<\theta \leqslant \theta_{c} ; \phi$ being constant $\mu_{c_{1}}>\mu_{c_{2}}$. Hence, with given thrust inclination, propellant mass ratio and initial mass, a greater thrust programme creates greater burnout velocity. Owing to the fourth equation of the set (15), and the second of (16),

$$
\begin{gathered}
X_{c_{1}}=\cos \delta \int_{1}^{\mu_{c}}\left[\log \mu / f_{1}(\mu)\right] \mathrm{d} \mu \text { and } X_{c_{2}}=\cos \delta \int_{1}^{\mu_{c}}\left[\log \mu \mathrm{d} \mu / f_{2}(\mu)\right] \\
\begin{aligned}
f_{1}(\mu)>f_{2}(\mu) \Rightarrow & (-\log \mu) / f_{1}(\mu)<(-\log \mu) / f_{2}(\mu) \\
& \Rightarrow \int_{\mu_{c_{1}}}^{1}(-\log \mu \mathrm{d} \mu) / f_{1}(\mu)<\int_{\mu_{2}}^{1}(-\log \mu \mathrm{d} \mu) / f_{2}(\mu) \\
& \Rightarrow \int_{1}^{\mu_{c_{1}}}(\log \mu \mathrm{d} \mu) / f_{1}(\mu)<\int_{1}^{\mu_{c_{2}}}(\log \mu \mathrm{d} \mu) / f_{2}(\mu) \\
& \Rightarrow X_{c_{1}}<X_{c_{2}}, \text { for } \mu_{c} \leqslant \mu<1,
\end{aligned}
\end{gathered}
$$

which shows that given the same three conditions, as mentioned earlier, the burnout range is less, though the overall range is greater, with greater thrust programme. For these two thrust programmes the second equation of (14) yields:

$$
h_{c_{1}}=-\int_{1}^{\mu_{c}}\left(\psi_{1} \mathrm{~d} \mu\right) / f_{1}(\mu) \text { and } h_{c_{2}}=-\int_{1}^{\mu_{c}}\left(\psi_{2} \mathrm{~d} \mu\right) / f_{2}(\mu), \quad\left(\mu_{c}<1\right),
$$

where $\psi_{1}$ and $\psi_{2}$, respectively, represent the corresponding instantaneous vertical velocities. Though $\psi_{1}>\psi_{2}, f_{1}(\mu)>f_{2}(\mu)$ does not necessarily imply $\psi_{1} / f_{1}(\mu)>\psi_{2} / f_{2}(\mu)$ 
and hence does not necessarily imply $h_{c_{1}}>h_{c_{2}}$; whether $h_{c_{1}} \geqslant h_{c_{2}}$ or $<h_{c_{2}}$ depends on the nature of the thrust law. In the cases of constant thrust $\left(T_{1}\right)$ and acceleration $\left(T_{2}\right)$ programmes where $f_{1}(\mu)=\tau_{i}$ and $f_{2}(\mu)=\mu \tau_{i}$, respectively,

$$
\psi_{1}=-\left[(1-\mu) / \tau_{i}\right]-\sin \delta \log \mu \text { and } \psi_{2}=\left[(\log \mu) / \tau_{i}\right]-\sin \delta \log \mu .
$$

Since $\log \mu=\log \{1-(1-\mu)\}=-(1-\mu)-\left[(1-\mu)^{2} / 2\right]-\ldots<-(1-\mu), \quad \psi_{1}>\psi_{2}$. This can also be proved otherwise, for $T_{1}>T_{2}$. Now,

$$
\begin{aligned}
\frac{\psi_{2}}{f_{2}(\mu)}-\frac{\psi_{1}}{f_{1}(\mu)}= & \frac{1}{\tau_{i}^{2}}\left(\frac{\log \mu}{\mu}+1-\mu\right)-\frac{\sin \delta \log \mu}{\mu \tau_{i}}(1-\mu), \\
\left(\text { as } \frac{\log \mu}{\mu}+1-\mu\right. & =\frac{1}{\mu}\left\{\left[-(1-\mu)-\frac{(1-\mu)^{2}}{2}-\ldots\right]+\mu(1-\mu)\right\} \\
& \left.=\frac{1}{\mu}\left[-\frac{3}{2}(1-\mu)^{2}-\frac{(1-\mu)^{3}}{3}-\ldots\right]=\text { negative quantity. }\right) \\
\frac{\psi_{2}}{f_{2}(\mu)}-\frac{\psi_{1}}{f_{1}(\mu)}= & \frac{1}{\mu \tau_{i}^{2}}\left[-\frac{3}{2}(1-\mu)^{2}+\tau_{i} \sin \delta\left\{(1-\mu)^{2}-\frac{(1-\mu)^{3}}{3 \tau_{i} \sin \delta}+\frac{(1-\mu)^{3}}{2}-\right.\right. \\
& \left.\left.-\frac{(1-\mu)^{4}}{4 \tau_{i} \sin \delta}+\frac{(1-\mu)^{4}}{3}-\ldots\right\}\right] \\
= & \frac{(1-\mu)^{2}}{\mu \tau_{i}^{2}}\left[\tau_{i} \sin \delta-\frac{3}{2}+\left(\frac{\tau_{i} \sin \delta}{2}-\frac{1}{3}\right)(1-\mu)+\right. \\
& \left.+\left(\frac{\tau_{i} \sin \delta}{3}-\frac{1}{4}\right)(1-\mu)^{2}+\ldots\right]
\end{aligned}
$$

This value (50a) is positive or negative depending on the values of $\tau_{i}, \delta$ and $\mu$, and is however found to be positive for $\tau_{i} \sin \delta \geqslant 3 / 2$ and $\mu<1$. Therefore, in this case $\psi_{2} / f_{2}(\mu)>\psi_{1} / f_{1}(\mu)$ is ensuing greater burnout altitude for the constant acceleration programme than for the constant thrust programme. When $\tau_{i} \sin \delta \leqslant 1$, the right hand side expression of $(50 a)$ becomes

$$
\begin{aligned}
& \leqslant\left[(1-\mu) /\left(\mu \tau_{i}^{2}\right)\right]\left[-\frac{1}{2}+\left(\frac{1}{2}-\frac{1}{3}\right)(1-\mu)+\left(\frac{1}{3}-\frac{1}{4}\right)(1-\mu)^{2}+\ldots\right] \\
& <\left[(1-\mu) /\left(\mu \tau_{i}^{2}\right)\right]\left[-\frac{1}{2}+\left(\frac{1}{2}-\frac{1}{3}\right)+\left(\frac{1}{3}-\frac{1}{4}\right) \ldots \infty \text { times }\right] \\
& <0, \Rightarrow \psi_{2} / f_{2}(\mu)<\psi_{1} / f_{1}(\mu), \text { for } \mu<1
\end{aligned}
$$

which due to (50) shows that higher burnout altitude is accomplished with the constant thrust programme than with the constant acceleration programme; of course in both the cases with the same thrust inclination, propellant mass ratio and initial mass, notwithstanding the fact (Maitra 1980) that for $\tau_{i} \sin \delta \leqslant 1$, the burnout position occurs below the horizontal plane of launching, or on it. Finally it is clear 
that higher inclination of thrust and initial thrust-to-weight ratio in this context leads to greater burnout altitude for the latter thrust programme than for the former.

To sum up, the results obtained in the foregoing analysis of a contrived flight are as follows. As an outcome of the present investigations, any arbitrary thrust programme, regardless of the law of thrust variation with respect to time or propellant mass ratio, entails analytical solutions to the differential equations of motion involved, followed by formulations of the range, endurance and final velocity (arising out of both powered and coasting flight) and that too in closed form subject to closed-form evaluation of the integral (23) denoted by $1 / K$. It has already been established that all these three have in the present situation greater values with greater thrust programmes without following exactly the same trend at the burnout position. The fact that the overall range, endurance and final velocity, the computation of which calls for the computation of only the value of $K$ concerned with different thrust laws, increase with the enhanced thrust programmes having the same thrust inclination, propellant mass ratio and lift-off mass, is attributable mainly to greater burnout velocity caused by the greater thrust programme. Hence, in the present frame work of rocket performance in a vacuum, the analytical solutions, in close form or otherwise to the overall performance are indeed peculiar to every thrust law coupled with a constant thrust inclination with respect to the horizon.

\section{List of symbols}

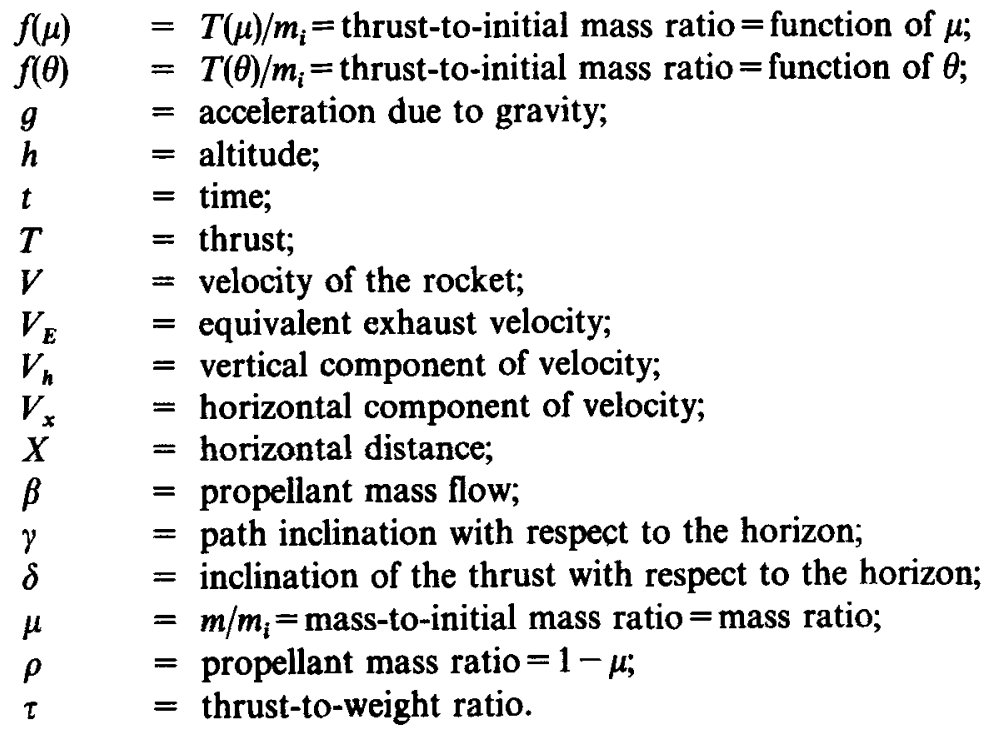

The dot sign indicates derivative with respect to time $t$. Subscript $i$ corresponds to the initial values of parameters whereas subscript $c$ is used for burnout parameters and subscript $f$ for final or overall parameters.

\section{References}

Maitra S N 1980 J. Aeronaut. Soc. India 32(1-4): 85-93

Miele A 1962 Flight mechanics (Reading, Mass: Addison-Wesley) vol. 1 